\title{
泵站运行中停电事故应急处理及预防措施
}

\author{
葛晓婷 \\ 新疆维吾尔自治区塔里木河流域巴音郭楞管理局博斯腾湖管理处 \\ DOI:10.32629/hwr.v4i2.2748
}

[摘 要] 本文通过对实际案例的分析, 了解原转运行中停电事故带来的影响,并在此基础上对应急处理及预防措施进行探讨分析,以保证原站运 行质量,减少事故的发生。

[关键词] 原站运行; 停电事故; 应急处理及预防措施

以某地区泵站为例, 泵站的总设计流量在每秒19.7立方米左右, 校核 流量约在每秒 15.6 立方米左右, 总的设计扬程为 12.86 米。该原站中进水流 道以矩形结构为主, 混流洜与喇叭口自流通道直接连接取水。另外, 在洜站 内设置了6台装机组, 其中主机组 4 台, 调节机组 2 台, 装机容量为 5500 千瓦。 百站运行采用 $35 \mathrm{KV}$ 变电站以同杆线路架设实现电能输送。

\section{1 断电问题应急操作}

1. 1 断电后的应急操作。正常情况下, 原站运行中出现断电问题后, 会 按照事故停运流程关闭相关闸门及阀门, 以免回流问题引发机组倒转。所 以在处理断电问题时, 需要先对闸门、阀门的关闭情况实行检查, 如果能正 常关闭, 即可在关闭后立即将故障问题上报调度中心。如果不能正常关闭, 要立即采取手动关闭的方式, 以此减少问题的产生, 并在关闭后上报调度 中心, 分析和解决故障, 减少损失及危险的形成。

1.2 原因查找。在应急操作完成后, 工作人员就要对断电问题产生的原 因进行查找, 在最短时间内解决断电问题, 恢复葲站的正常运转。通常情况 下, 断电原因的产生与 $2 \mathrm{~B} 、 3 \mathrm{~B}$ 主变高压侧及 $35 \mathrm{KV}$ 进线侧有着接关系, 人员要 仔细检查这些区域有无带电显示, 并对 $35 \mathrm{KV}$ 进线电压实行检测, 查看其是 否在标准范围值内。在检查过程中, 如果 $2 \mathrm{~B} 、 3 \mathrm{~B}$ 主变高压侧及 $35 \mathrm{KV}$ 进线断 路器均未出现跳闸现象, 且保护装置也并未存在报警现象, 则可检查主机 组断路器, 如果发现主机组断路器出现报警现象, 则说明是 $35 \mathrm{KV}$ 线路出现 故障问题导致的断电, 确定断电原因后, 及时连接调度中心。

\section{2 蜃站内各设备的断电操作}

在断电问题发生后, 为增强洜站内其他机电设备的安全性, 需要对机 电设备均实施断电操作。泵站内的机电设备有: $400 \mathrm{~V}$ 低压设备、35KV、10KV 高压开关柜设备、35KV高压柜手推设备、35kv进线隔离刀闸等, 断电操作 分别为:

(1) $400 V$ 低压设备分闸处理。先断开42B变压器, 利用手摇方式将 $42 B$ 断路器摇到试验位置, 之后将 $400 \mathrm{~V}$ 低压室中抽屉柜内的旋转按钮扭转到分 闸位置上。

(2) $35 \mathrm{KV} 、 10 \mathrm{KV}$ 高压开关柜分闸处理。10KV高压开关柜分闸时, 先将两 台主变高低压变压器实施分闸处理, 转换开关要调到就地位置, 之后转动 开关到分闸位置后松开, 确定分闸效果。35KV高压开关柜分闸时, 分别将进 线、母联开关、35KV室分别予以分闸处理, 之后按照 $10 \mathrm{KV}$ 分闸流程完成操 作, 确认分闸效果。

(3) $35 \mathrm{KV}$ 高压柜手推设备断电操作。首先, 查验开关柜位置, 确保其 在分闸位置上; 其次, 将手车摇把插入开关柜下方摇把口内, 在插入前先 将保护片拨开, 之后按照逆时针方向转动摇把, 将手车移动到指定位置。 通过对显示屏的观察来判断设备断开情况, 完全断开后, 拔出摇把, 完成 作业。

(4) $35 \mathrm{kv}$ 进线隔离刀闸的断电操作。先对高压开关柜的分闸情况、手
车的推出情况予以确定, 确保其处在分闸及全部推出状态下, 打开隔离闸 的锁链, 在此过程中, 工作人员要佩戴安全防护设施。隔离闸锁链打开后, 向下搬动隔离闸, 达到分闸效果。

\section{3 送电操作}

断电故障解决完成后, 需要实行供电试验, 恢复洜站的正常运行。在送 电中, 工作人员要严格按照上级指令要求, 逐步开始送电作业, 避免电流突 然增大带来不良影响。在送电操作中, 先要合起 $35 \mathrm{KV}$ 进线隔离开关。与进 线隔离刀闸分闸作业相似, 工作人员应按照工作流程开展合闸作业。之后 实施母线合闸操作。母线进线柜的断路器手车要到指定位置, 并将开关搬 到就地位置上, 转换开关实施合闸操作, 显示器工作证明合闸成功。

随后开展主变高低压侧舍闸操作。与分闸流程类似, 不过在手车摇把 转动时, 要按照顺时针的方向转动, 待摇把转至规定位置后, 将转换开关打 至就地位置。最后, 实施 400 低压设备合闸。在确定摇把及转动开关在指定 位置后, 将转动开关转动到合闸位置后断开, 调整 $400 \mathrm{~V}$ 室的旋转按钮, 完成 合闸作业。

\section{4 断电期间注意事项}

在不清楚断电原因前提下, 应对直流屏、UPS蓄电池的能耗进行控制, 将相关设备电源开关切断, 关闭计算机等控制系统, 打开LCU柜柜门。在断 电后如果不能及时送电, 且时间间隔已经超过一天后, 需要对直流屏及蓄 电池充电, 并且检查空气开关状态, 确保其处于分闸状态。必要时可将 $400 \mathrm{~V}$ 室中生活区抽屉柜实施合闸处理, 以此为其提供临时电源供应。

\section{5 断电危害及预防措施}

5. 1危害。泵站在突然断电后, 机组设备会立即停止工作, 这时水池中 的水会逆流回管道中, 导致机组设备倒转。突然断电会导致控制系统中PLC 数据的丢失或损毁, 阻碍机组的自动化运转, 出现城市用水难题。

5.2 预防措施。为避免断电带来的不良影响, 需制定科学合理的预防方 案, 加大巡检力度, 及时监测接地电阻参数。再者, 做好值班人员轮换及交 接作业, 准确了解机组运行情况, 防止各类问题的产生。

\section{6 结语}

原站运行中的断电问题, 对于原站机组的运行安全有着直接影响, 人 员应制定合理的应急及预防方案, 科学处理断电问题, 以此减少损失及危 险的形成, 提高洜站的运行质量。

\section{[参考文献]}

[1]张琪,王琛.泉站运行中停电事故应急处理及预防措施[J]. 山东水 利,2018,(10):36-37.

[2]买买提 - 阿不都热衣木. 硫化氢中毒事故预防与应急处理措施 [J]. 安全、健康和环境,2007,(07):35-36.

[3]郑伟, 丁辉明,刘国栋, 等.排水百站运行管理设计与应用 [J].净水技 术,2019,38(S2):94-98. 